\title{
Design of Circularly Polarized Multi-frequency Microstrip Antenna
}

\author{
Yanzhong $\mathrm{Yu}^{*}$, Rongjiao Li and Mengya Lin \\ College of Physics \& Information Engineering, Quanzhou Normal University, Quanzhou, 362000, China \\ ${ }^{*}$ Corresponding author
}

\begin{abstract}
This paper presents a dual circular polarization microstrip antenna with different sizes of two square patches stacked, so that it can realize dual-band antenna. Right circular polarization in $\mathrm{S}$ band is achieved by the upper patch antenna with diagonal corner cut method and with single feed method. The lower layer antenna patch uses the surface open-u-shaped slot for the left circular polarization and miniaturization of the $L$ band. And then by HFSS14.0, the antenna has a gain of $4 \mathrm{~dB}$ in Right circular, and has good performance in the S-band suppression cross-polarization and anti-multipath interference.
\end{abstract}

Keywords-microstrip antenna; circular polarization; miniaturization

\section{INTRODUCTION}

The global positioning systems have the United States GPS system, China's Beidou Navigation System (BDS), Russia's GLONASS, and Europe's Galileo. But BDS is a kind of positioning system researched and developed by china and it encompasses most of China and its neighboring countries.

Beidou system applies to military and civilian, but mainly for civilian. Beidou satellite navigation system plays an important role in location services of personal and vehicle, management of road and rail traffic, land transport, water transport, air transport, geological disaster monitoring, emergency rescue, power time synchronization system, and so on.

All the time, the shape of the microstrip antenna patch, feeding technology, and other aspects become the main research topics. There is not only making some progresses in theory, but also expanding application. Microstrip antenna has many advantages, such as simple structure, light weight, low profile, easier to implement circular polarization, integrated with active devices, carrier conformality and processing.

We are familiar with several circular polarization methods as (a) cutting angle; (b) the slotted of the surface; (c) quasi-square, nearly circular, nearly equilateral triangle; (d) with tuned branches and so on.

The circular polarization of the proposed antenna can be realized by using the cut angle method and the slotted of the surface method. That is to say, the upper layer of the diagonal cut off two isosceles triangles, the lower surface of the patch to decrease a u-shaped groove, unequal of Left and right arm lengths, and the left circular polarization is achieved by feeding with the single feed method.

\section{ANTENNA DESIGN}

The basic geometry of the proposed antenna is shown in Figure 1. In this letter, the antenna is a stacked structure composed of two square radiation patches with different areas. To make a better right-circular polarization, it is through the way and using the single feed method. The lower patch is to realize a left circular polarization and miniaturization by opening a U-shaped groove with an asymmetrical arm length on the surface of the radiation patch. At the same time, the upper and lower antenna patches are filled with dielectric substrates having different dielectric constants and thicknesses. The right circular polarization of the antenna can usually be achieved by cutting isosceles right triangle with side length a that is the upper antenna patch of the antenna, as shown in Figure 2. And then it adopts the coaxial feed to achieve circular polarization as illustrated in Figure 3. When left circular polarization will occur. We can use a U-shaped slot with a left arm longer than the right arm to achieve the left circular polarization of the antenna. The specific structure is depicted in Figure 4. The dielectric constant of the dielectric substrate is proportional to the square of the antenna frequency. Therefore, in this design, the lower dielectric substrate is a Rogers RO3006 with a dielectric constant of 6.15 . For the upper layer, the antenna uses FR4_epoxy with a dielectric constant of 4.4.

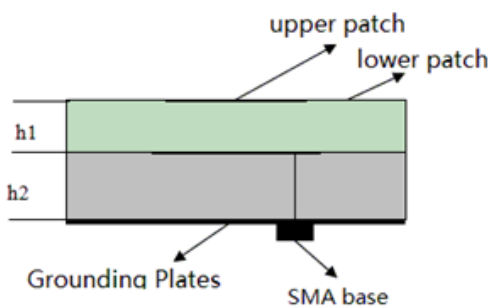

FIGURE I. SIDE VIEW FOR THE PROPOSED ANTENNA

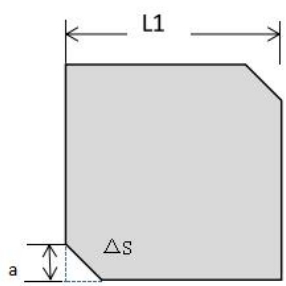

FIGURE II. UPPER ANTENNA PATCH 


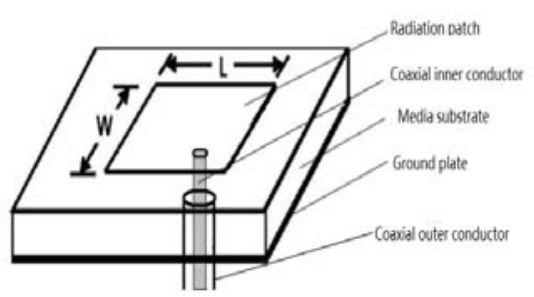

FIGURE III. COAXIAL FEED

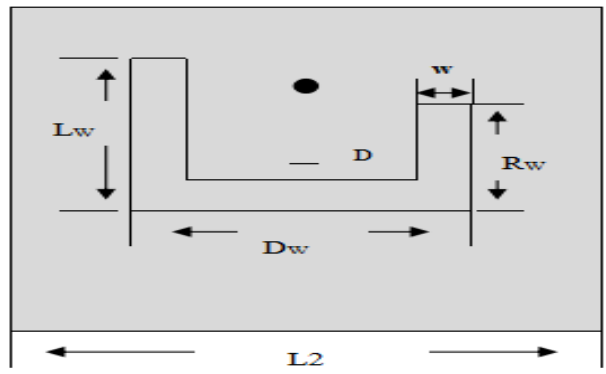

FIGURE IV. LOWER ANTENNA PATCH

In order to make more convenient, some variables are defined in the design. the length of the upper radiating patch is $\mathrm{L1}$; the edge length of the small triangle at the diagonal is a; the height of the upper medium is hl; the length of the coaxial feed is feed $\mathrm{h}$; the edge length of the lower radiating patch is L2; the left arm of the U-shaped groove is Lw; the right arm is Rw; the length of the bottom end groove is Dw; the width of the groove is $\mathrm{w}$; the distance from the bottom of the groove to the center of the patch is $\mathrm{D}$; the distance from the feed point to the center is feed y; and the thickness of the lower medium substrate is $\mathrm{h} 2$. The specific data is shown in the table.1.

TABLE I. PARAMETERS FOR THE DESIGNED ANTENNA

\begin{tabular}{|c|c|c|c|}
\hline parameter & Value $(\mathrm{mm})$ & parameter & value $(\mathrm{mm})$ \\
\hline $\mathrm{L} 1$ & 25.6 & $\mathrm{Lw}$ & 11 \\
\hline $\mathrm{a}$ & 6.36 & $\mathrm{Dw}$ & 6 \\
\hline $\mathrm{h} 1$ & 1.4 & $\mathrm{w}$ & 1.3 \\
\hline feed_h & 15 & $\mathrm{D}$ & 4.83 \\
\hline $\mathrm{L} 2$ & 33.3 & $\mathrm{~h} 2$ & 2.3 \\
\hline $\mathrm{Rw}$ & 8.4 & feed_y & 3.2 \\
\hline
\end{tabular}

\section{Simulation Results}

The antenna is simulated by HFSS. In the L-band, as shown in Figure 5, the antenna has an S11 value of $-12.93 \mathrm{~dB}$ at 1.612 $\mathrm{GHz}$ and a bandwidth of $42 \mathrm{MHz}(1646 \mathrm{MHz}-1604 \mathrm{MHz})$, which is consistent with the design requirements. In Figure 6, when $\mathrm{Lc}=3.2 \mathrm{~mm}$, the input impedance at the operating frequency of $1.615 \mathrm{GHz}$ is $(76.68-\mathrm{j} 3.733) \Omega$. From Figure 7 , the normalized impedance at $1.616 \mathrm{GHz}$ is $1.626-\mathrm{j} 0.0786 \Omega$, and the impedance matching is good. From Figure 8, when the frequency is $1.616 \mathrm{GHz}$, the axial ratio reaches a minimum value of $0.93 \mathrm{~dB}$. Figure 9 show that the antenna has a strong performance to suppress cross-polarization and anti-multipath interference in the L-band. It is demonstrated that the antenna is implemented with L-polarization at the L-band, and the polarization performance is good, which meets the design requirements.

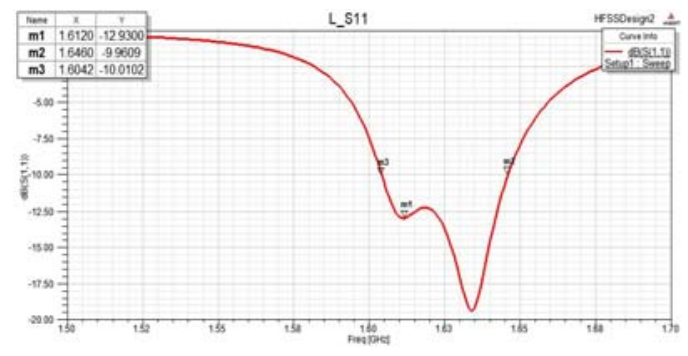

FIGURE V. S11 FOR THE DESIGNED ANTENNA

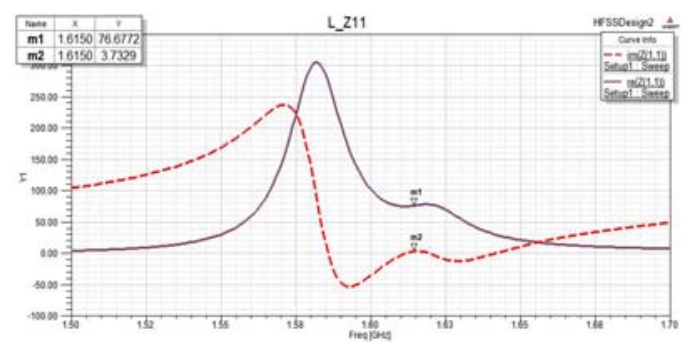

FIGURE VI. INPUT IMPEDANCE VS FREQUENCY

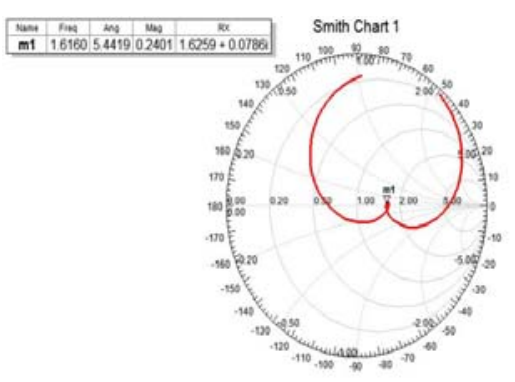

FIGURE VII. SMITH CHART RESULT OF S11

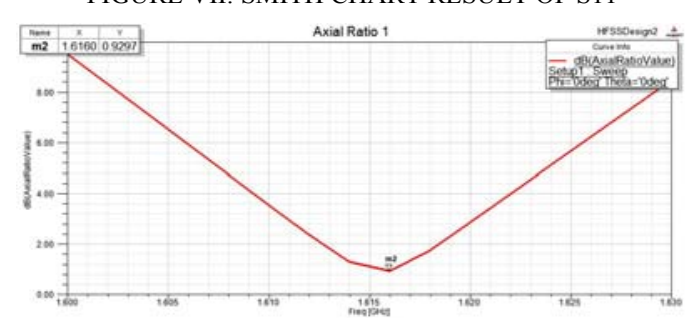

FIGURE VIII. AXIAL RATIO

In S-band, the designed microstrip antenna has an S11 value of $-21.647 \mathrm{~dB}$ at $2.491 \mathrm{GHz}$ and a bandwidth of 110 $\mathrm{MHz}$, which is in accordance with the design requirements, as shown in Figure 10. Figure 11 shows the input impedance of the antenna at $2.493 \mathrm{GHz}$ is $40.452-\mathrm{j} 0.016 \Omega$. In Figure 12, with a frequency of $2.492 \mathrm{GHz}$, the normalized impedance is $0.8491-\mathrm{j} 0.0017 \Omega$, so that indicating good matching is achieved at this operating frequency point. Figure 13 shows that the axial ratio at $2.491 \mathrm{GHz}$ is $0.461 \mathrm{~dB}$. The axial ratio at a frequency of $2.494 \mathrm{GHz}$ reaches a minimum of $0.158 \mathrm{~dB}$. It can be seen from Figure 14 (a) and (b) that the right-handed gain of the antenna reaches to $4 \mathrm{~dB}$. And the RHCP level is higher than the LHCP level, and more than $15 \mathrm{~dB}$. It indicates that the antenna in the $\mathrm{S}$ band suppression cross polarization and antimultipath interference is very strong. 

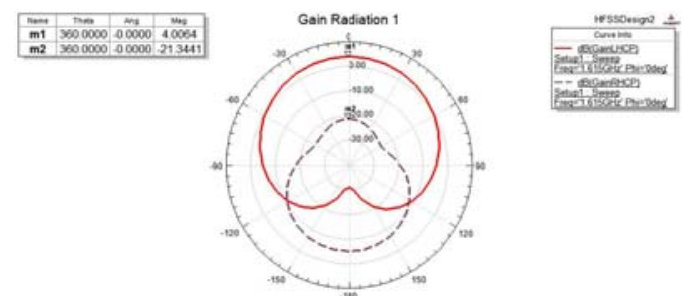

(a)

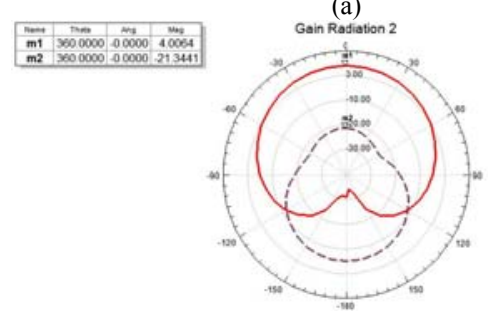

(b)

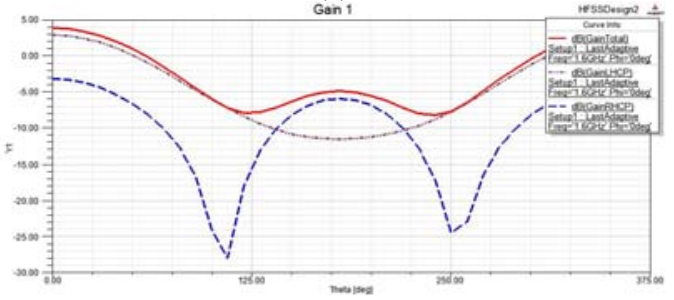

$(\mathrm{c})$

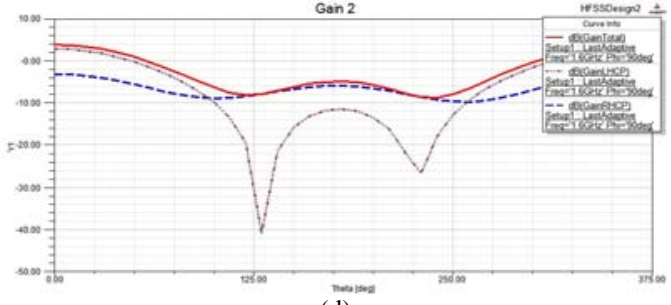

(d)

FIGURE IX. GAIN PATTERN OF THE XZ AND YZ CROSS SECTIONS OF THE ANTENNA IN THE L BAND

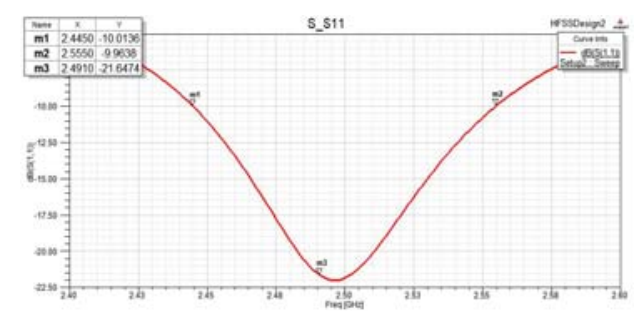

FIGURE X. S11 SWEEP RESULT ANALYSIS

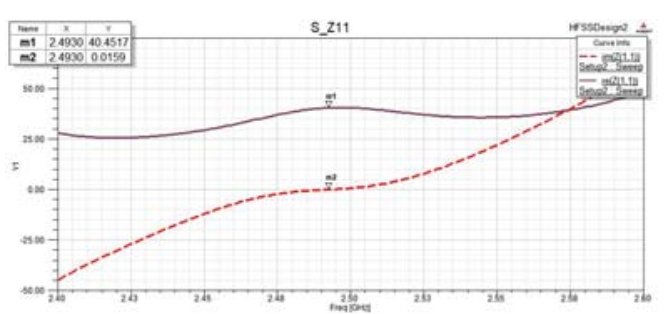

FIGURE XI. INPUT IMPEDANCE AS A FUNCTION OF FEED POINt

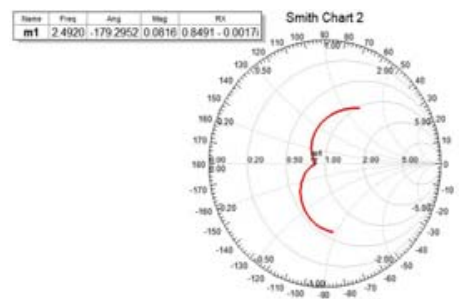

$=x^{2}$

FIGURE XII. SMITH CHART RESULTS FOR S11

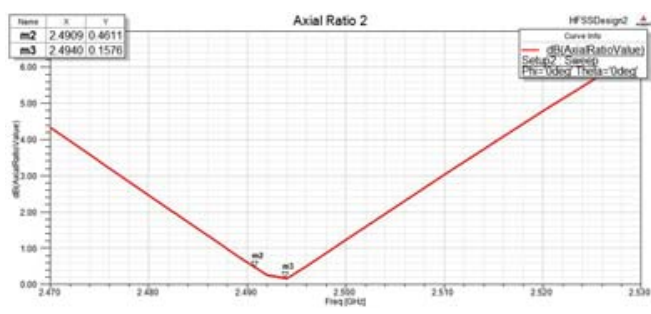

FIGURE XIII. AXIS RATIO SWEEP ANALYSIS RESULTS
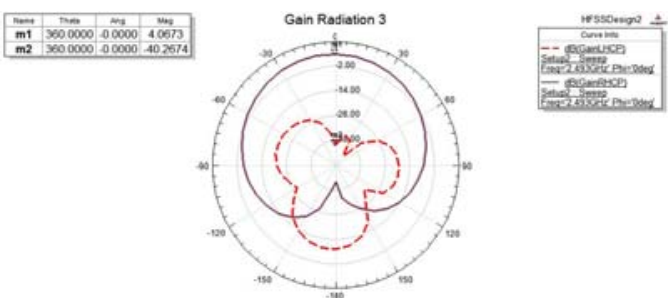

(a)
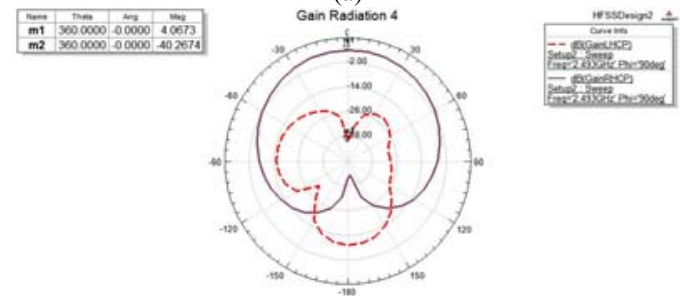

(b)

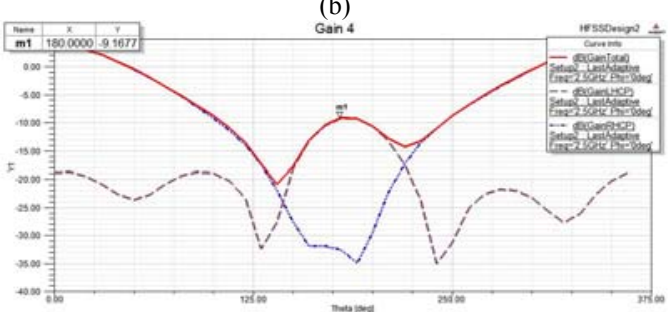

(c)

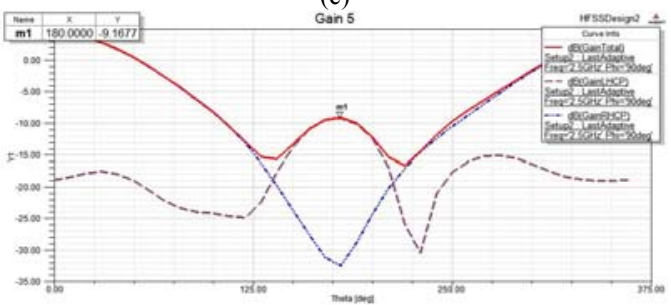

(d)

FIGURE XIV. GAIN PATTERNS OF THE XZ AND YX SECTIONS OF THE S BAND 


\section{CONCLUSION}

In this paper, the design of this antenna is based on the actual needs of the antenna. To achieve dual-band operation and reduce the antenna size, a circularly polarized microstrip antenna model based on a double-layer square patch structure is presented. The frequency band is in the S-band and L-band of the BDS system. This design is not only satisfies of the requirements of the antenna on both frequency bands, but also the antenna is smaller in size, simpler in structure, and less expensive than other antennas. This is extremely important for the promotion and use of Beidou equipment.

\section{ACKNOWLEDGEMENT}

This work is sponsored by the Quanzhou High-level Talent Innovation and entrepreneurship project (2017G050) and the Fujian Province Project of Innovative Entrepreneurship of Undergraduate (201710399046).

\section{REFERENCES}

[1] Bang J H, Enkhbayar B, Min D H, et al. A Compact GPS Antenna for Artillery Projectile Applications [J]. IEEE Antennas \& Wireless Propagation Letters, 2011, 10(1):266-269.

[2] Sun X, Fan C, Zeng G, et al. A novel compact planar multi-band antenna for GPS, WLAN and WIMAX applications [C]. Microwave Conference Proceedings. IEEE, 2011:1-3.

[3] Lorenzo D S D, Lo S C, Enge P K, et al. Calibrating adaptive antenna arrays for high-integrity GPS [J]. Gps Solutions, 2012, 16(2):221-230.

[4] Wei K, Zhang Z, Feng Z. Design of a Coplanar Integrated Microstrip Antenna for GPS/ITS Applications [J]. IEEE Antennas \& Wireless Propagation Letters, 2011, 10(1):458-461.

[5] Chen S C, Liu G C, Chen X Y, et al. Compact Dual-Band GPS Microstrip Antenna Using Multilayer LTCC Substrate [J]. IEEE Antennas \& Wireless Propagation Letters, 2010, 9(1):421-423.

[6] Ding X. Development of BeiDou Navigation Satellite System [J]. Proceedings of International Technical Meeting of the Satellite Division of the Institute of Navigation, 2011.

[7] Xie J, Liu T. Research on Technical Development of BeiDou Navigation Satellite System[J]. 2013, 243:197-209.

[8] Chang K. Compact and Broadband Microstrip Antennas [M]. 2002. 